\title{
Upper extremity deep vein thrombosis (UEDVT); Does end stage renal disease (ESRD) triggers UEDVT?
}

\author{
MN Qureshi* \\ Consultant Emergency Medicine, King Faisal Specialist Hospital \& Research Centre, Riyadh, Saudi Arabia
}

A 50 years old man, presented to the Emergency department, with progressive left arm swelling, which started few days ago. He was a known case of ESRD (secondary to lupus nephritis), on dialysis for the past five years. He had a right sided subclavian catheter, for dialysis. He had no surgical interventions or injuries, to his left upper extremity. He was apyrexial, hemodynamically stable, with significant edema and erythema over his left upper extremity. There were no signs of limb ischemia. Ultrasound followed by venography of the involved extremity, revealed venous thrombosis of limb veins, which extended to the left subclavian and internal jugular vein. The patient was successfully treated with anticoagulation therapy, in an outpatient setting. There were no further episodes of venous thromboembolism (VTE), at 6 months follow up. There has been no similar case reports in published literature (Figure 1).

\section{Introduction}

Up to $10 \%$ of all deep vein thrombosis (DVT) are related to upper extremities, with an incidence of, 3 per 100,000 in the general population [1-3]. The incidence of UEDVT is less than the lower extremity DVT, possibly due to following factors [4-6].

- Lesser venous valves in the upper extremity.

- bedridden patients generally have more arm than leg movements,

- less hydrostatic pressure in the arms,

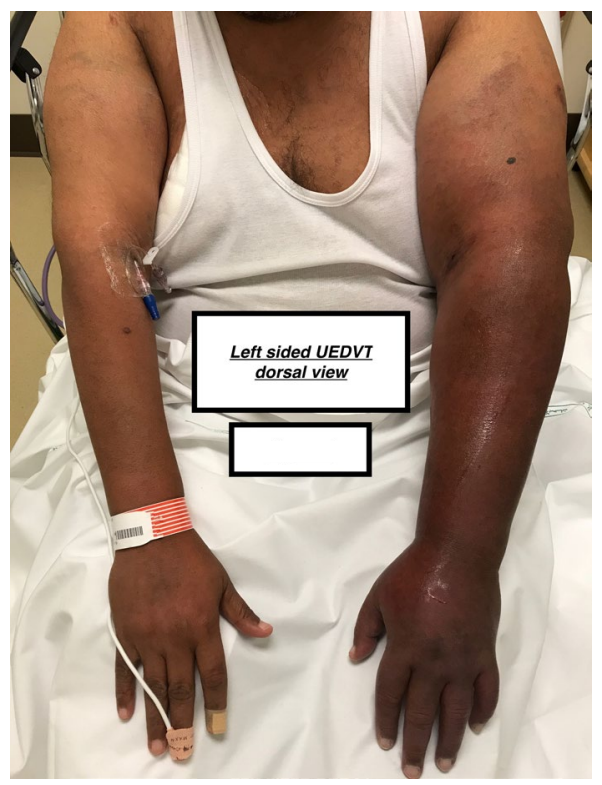

Figure 1. Left sided UEDVT dorsal view
- fibrinolytic activity is more, in the vascular endothelium of the arms,

- Absence of a complex network of veins, as seen in the calf muscles.

The incidence of thrombosis in the upper extremity is maximal, in the subclavian vein (18-67\%), followed by axillary $(5-25 \%)$ and the brachial (4-11\%), with marked predilection for the left side, probably as a result of anatomical reasons [7]. UEDVT involves more than one segment of the vein, at a time. Venous catheters are mostly implicated with UEDVT [8]. Larger the size of the inserted venous catheter, higher is thought to be the chance of clot formation [9]. Other factors are also thought to play an important role, but the information regarding the whole spectrum of UEDVT is still lagging [10].

We wanted to explore, the pathophysiology, incidence, diagnostic techniques and management of UEDVT, in general population \& specifically within the ESRD patients (Figure 2).

\section{Search strategy}

We did a literature search using PubMed central (PMC), through the National Centre of Biotechnology information website (NCBI).

Key words: Upper extremity, Deep vein thrombosis, End stage renal disease.

A total of 977 articles were retrieved. After reviewing the abstracts, 66 articles were found to be relevant, which have been referenced.

\section{The search strategy has been described below:}

((("upper"[All Fields] AND "extremity"[All Fields] AND "deep"[All Fields] AND "vein"[All Fields] AND "thrombosis"[All Fields]) OR "upper extremity deep vein thrombosis"[All Fields]) OR ("upper extremity deep vein thrombosis"[KYWD] OR ("upper"[All Fields] AND "extremity"[All Fields] AND "deep"[All Fields] AND "vein"[All Fields] AND "thrombosis"[All Fields]) OR "upper extremity deep vein thrombosis"[All Fields])) AND (("kidney failure, chronic"[MeSH Terms] OR ("kidney"[All Fields] AND "failure"[All Fields] AND "chronic"[All Fields]) OR "chronic kidney failure"[All Fields] OR ("end"[All Fields] AND "stage"[All Fields] AND "renal"[All Fields] AND "disease"[All Fields]) OR "end stage renal disease"[All Fields])

${ }^{*}$ Correspondence to: Muhammad Nauman Qureshi, Consultant \& Research Director, Department of Emergency Medicine, King Faisal Spe-cialist Hospital \& Research Centre, Riyadh, Saudi Arabia, E-mail: qmuhammad@kfshrc.edu.sa

Key words: magnetic resonance, $3 D$ - SPACE, cortical malformations, schizencephaly

Received: August 26, 2020; Accepted: October 19, 2020; Published: October 22,2020 


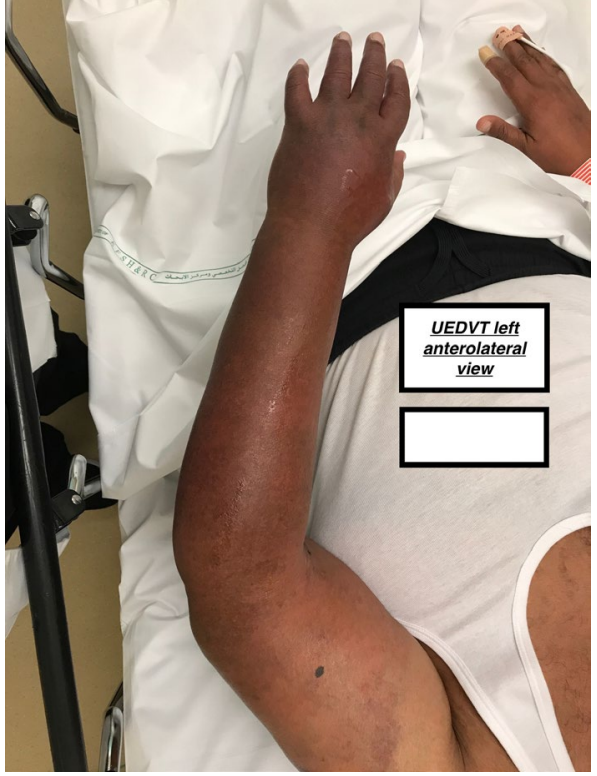

Figure 2. UEDVT left anterolateral view

OR ("kidney failure, chronic"[KYWD] OR ("kidney"[All Fields] AND "failure"[All Fields] AND "chronic"[All Fields]) OR "chronic kidney failure"[All Fields] OR ("end"[All Fields] AND "stage"[All Fields] AND "renal"[All Fields] AND "disease"[All Fields]) OR "end stage renal disease"[All Fields]))

\section{Pathophysiolog}

"Virchow's Triad" is a multifactorial phenomenon, that leads to thrombosis [11]. Venous stasis, vascular injury and hyper coagulability are the three factors, which work singly or together, to generate a thrombus [12]. DVT starts within the venous valves, of the deep veins of the leg. Venous thrombi tend to form in the sinuses, adjacent to venous valves $[13,14]$.

Venous stasis decreases oxygen tension, which increases hematocrit [15]. This leads to expression of some procoagulants (e.g p-selectin), which attracts tissue factor and downgrades certain anticoagulant proteins (like thrombomodulin), which are expressed on venous valves [16]. The risk of thrombus formation increases proportionately, as the ratio between the procoagulants and anticoagulant factors is disturbed [17].

In some patients, genetic variants lead to higher levels of certain procoagulant factors (VII, VIII, IX and prothrombin), which increases the risk of thrombus formation. Malignancy can exert a constrictive effect on veins, leading to stasis. It also sheds procoagulants, such as tissue factor on membrane particles that forms a thrombus [18].

Advancing age is associated with increased frequency of illness, prolonged immobility, co-morbid medical conditions and an increased level of procoagulant [19]. Paget Schroetter disease is characterised by UEDVT, induced by vigorous effort of the involved extremity, clavicular impingement and a sequelae of thoracic outlet syndrome [20].

ESRD is associated with increased procoagulants, like cystatin C, interleukin-6, tumor necrosis factor, intercellular adhesion molecule-1, fibrinogen and factor VIII. Anticoagulants, like protein C, protein S and anti-thrombin are comparatively decreased. The risk for thrombosis in patients with ESRD is further increased, as a result of platelet activation, occurring within the extracorporeal hemodialysis device. These factors lead to increase the risk of thrombosis in these patients, independent of the other conventional risk factors. Systemic lupus erythematosis (SLE), which leads to lupus nephritis and consequentially ESRD, is an additional factor, that can also enhance the risk of thrombosis in these patients [21].

\section{Classification}

Coon and Willis classified UEDVT, into two divisions; traumatic, which may be internal (caused by central venous cannulation) or external (like fracture, stress) and spontaneous (associated with cancer or idiopathic) [22].

Others have classified it as, primary (idiopathic, thoracic outlet syndrome, Paget-Schroetter syndrome) and secondary (venous catheter, cancer, surgery related) [23].

\section{Clinical presentation}

UEDVT can be asymptomatic. The typical presentation is, the presence of arm discomfort, edema, discolouration and dilated venous collaterals [24]. The level of suspicion increases in the presence of risk factors, like indwelling catheters and vigorous arm exercise (Figure 3).

\section{Diagnosis}

The diagnostic work up of DVT of the lower extremities is well established, however, the same is not true, in relation to UEDVT. A systematic review, throws light on the diagnostic values of various methods [25]. Ultrasonography (USG) is probably the first choice for investigating UEDVT and venography is used, when there is disparity between USG and clinical findings. It is also not known, whether the validated recurrent thrombosis "risk prediction models" for lower limb DVT (like the Vienna prediction model), which use a combination of clinical and diagnostic criteria, can be applied to patients with UEDVT [26,27].

\section{Management}

The options include anticoagulant therapy, thrombolysis \& thrombectomy (Figure 4).

\section{Anticoagulant therapy}

Anticoagulation is the mainstay of treatment, with the goal of re-canalising the thrombosed vein, preventing progression to pulmonary embolism (PE) and recurrence of thrombosis [28,29]. The recommendations for lower extremity DVT are applied to UEDVT.

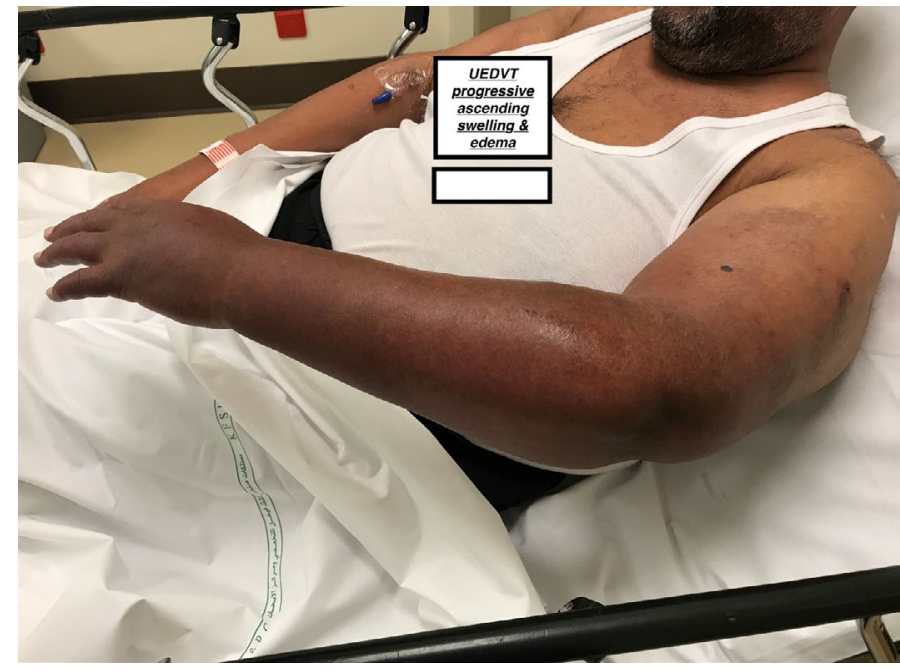

Figure 3. UEDVT progressive ascending swelling \& edema 


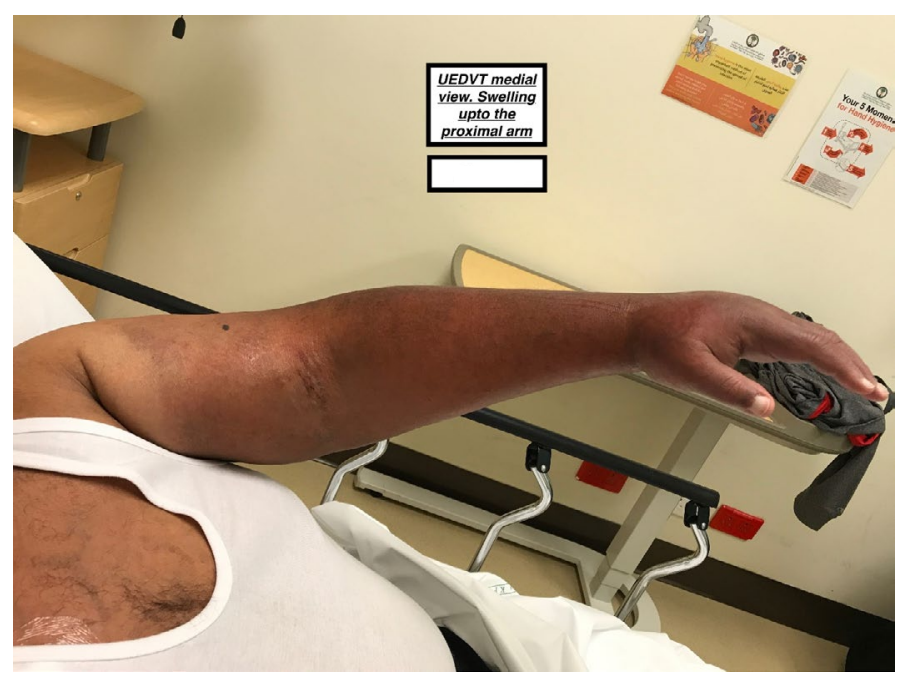

Figure 4. UEDVT medial view, swelling upto the proximal arm

Low Molecular Weight Heparin (LMWH) is preferred over unfractionated heparin (UFH), for treatment of thrombotic events. The predictability of their anti thrombotic effect, better compliance and absence of thrombocytopenia, favours them over heparin [30-35]. LMWH are overlapped with Vitamin K antagonists (like warfarin), to achieve an International Normalised Ratio (INR), in a range of 2.5-3.0.

Direct Oral Anticoagulants (DOACs), like dabigatran are also considered an important modality of treatment. Their safety and efficacy profile is not very clear in ESRD patients, as $80 \%$ of their clearance happens in the kidneys. The guidelines by American college of Chest Physicians (ACCP), recommend DOACs for all non-cancer related VTE and LMWH for VTE, associated with cancer [36].

Cohort studies indicate that the optimum duration of anticoagulation is for a period of 3-6 months [37,38]; however, the evidence is moderate. The current guidelines from ACCP recommend 3 months treatment, for unprovoked DVT.

However, multimodal therapy is recommended in patients, who present with massive DVT (massively swollen limb, acrocyanosis, severe pain, ischemia). These patients are more than twice as likely, to develop extension of thrombosis, compared to patients with less extensive DVT (11.2\% vs. $5.3 \%)$ [39,40].

\section{Systemic Thrombolysis (ST)}

Systemic thrombolysis, for fully obstructed segments of veins with DVT, does not have as much therapeutic efficacy, as seen in coronary \& cerebral thrombolysis. This is due to inefficient diffusion of the thrombolytic agent in the large venous thrombi, along with low flow conditions [41-43]. This approach, as compared to anticoagulation alone, is also associated with a very high risk of bleeding related complications [44-50].

\section{Catheter Directed Thrombolysis (CDT)}

It is a minimally invasive technique, which involves infusing a fibrinolytic agent directly in the thrombus, via a catheter, within the involved vessel. A 3-point scale has been proposed, to define the outcomes of this therapy. Grades 2 and 3, signify at least $50 \%$ luminal patency, which is classed as a satisfactory therapeutic outcome [51]. CDT has been compared with combined anticoagulation and compression stockings treatment, in patients with lower extremity
DVT. Immediate grade 2 to 3 lysis was observed in $40 \%$ of patients post procedure, with almost double the patency rates, in the former group. Absolute risk reduction of $28 \%$ (95\% CI 9.7-46.7\%; P < 0.004) was achieved [52]. This trial claimed superior clot lysis with CDT, with better long-term venous patency rates in patients with lower limb DVT. CDT is only considered selectively for treatment of UEDVT.

\section{Thrombectomy (THB)}

$\mathrm{THB}$, is one of the approved therapies, in the management of acute DVT of the lower limbs. The risk of post-thrombotic symptoms were less frequent in the THB group, when compared with anticoagulation group ( $7 \%$ vs. $42 \%$; $\mathrm{P}<0.005$ ). The luminal patency rates in the THB group were also better (76\% vs. 35\%; P < 0.025) [53]. THB applicability is not well established in UEDVT.

\section{Discussion}

UEDVT is a fatal disease, which is relatively under diagnosed [54]. Majority of the risk factors, which cause DVT of the lower extremities, also contribute in development of UEDVT. The risk factors also have a synergistic effect [55].

ESRD, increases the risk of DVT, due to imbalance between the pro and anti coagulants factors [56]. The insertion of central venous catheter and creation of arteriovenous fistulas play a significant part, in triggering VTE in an ESRD patient [57].

The prevalence of pulmonary embolism, has been reported at autopsy in patients dying of ESRD [58]. A prospective study conducted in an intensive care unit, found ESRD as a risk factor for DVT (hazard ratio (HR) 3.7, 95\% confidence interval (CI) 1.2-11.1) [59]. Lupus nephritis can have a contributory role, in development of VTE in ESRD patients, due to the systemic effect of the disease [60].

In our patient, there was no prior surgical or vascular intervention, in the affected upper extremity. In fact, the contralateral limb had a previous non-functional arteriovenous fistula and a central venous catheter. It is unclear, why the thrombosis occurred in an extremity, where there was seemingly, no vascular stasis or injury. It is likely to be related with the relative hyper-coagulable state, in ESRD patients, with possible synergistic effect from the SLE [61].

PE complicates UEDVT in $36 \%$ of cases and is more commonly associated with central venous catheters [62]. Post-thrombotic syndrome, which occurs in up to $50 \%$ of patients (within 2 years of DVT), has similar prevalence in UEDVT [63]. The mortality rate in UEDVT patients, range from 10 to $50 \%$, mainly due to the underlying malignancy $[64,65]$.

The 30 -day mortality rate exceeds $3 \%$ in patients with DVT, who are not anti-coagulated, and this mortality risk increases 10 -fold in patients who develop PE.

The current scientific research is unclear, about the use of prophylactic anticoagulants in ESRD patients.

\section{Conclusions}

High index of suspicion is required in patients with ESRD, to diagnose UEDVT. Prevention strategies for UEDVT in ESRD patients can be explored further.

\section{References}

1. Coon, William W., and Park W. Willis. "Thrombosis of axillary and subclavian veins." Archives of surgery 94, no. 5 (1967): 657-663. 
2. Heil, Jan, Wolfgang Miesbach, Thomas Vogl, Wolf O. Bechstein, and Alexander Reinisch. "Deep vein thrombosis of the upper extremity: a systematic review." Deutsches Ärzteblatt International 114, no. 14 (2017): 244.

3. Horattas, Mark C., Dennis J. Wright, Andrew H. Fenton, D. M. Evans, M. A. Oddi, R. W. Kamienski, and E. F. Shields. "Changing concepts of deep venous thrombosis of the upper extremity--report of a series and review of the literature." Surgery 104, no. 3 (1988): 561-567

4. Gross, C. M. "Gray's anatomy, Anatomy of the Human Body. 28th American ed." Philadelphia: Lea and Febiger (1966): 730.

5. Pandolfi, M., B. Robertson, S. Isacson, and Inga Marie Nilsson. "Fibrinolytic activity of human veins in arms and legs." Thrombosis and Haemostasis 20, no. 02 (1968): $247-256$.

6. Robertson, B. R., ROBERTSON BR, and NILSSON IM. "Response of local fibrinolytic activity to venous occlusion of arms and legs in healthy volunteers." (1972).

7. Hill, Stephen L., and Robert E. Berry. "Subclavian vein thrombosis: a continuing challenge." Surgery 108, no. 1 (1990): 1.

8. Saseedharan, Sanjith, and Sunil Bhargava. "Upper extremity deep vein thrombosis." International journal of critical illness and injury science 2, no. 1 (2012): 21.

9. Marinella, Mark A., Satheesh K. Kathula, and Ronald J. Markert. "Spectrum of upperextremity deep venous thrombosis in a community teaching hospital." Heart \& lung 29 , no. 2 (2000): 113-117.

10. Prescott, STEPHEN M., and G. E. R. A. S. I. M. Tikoff. "Deep venous thrombosis of the upper extremity: a reappraisal." Circulation 59, no. 2 (1979): 350-355.

11. Grove, Jay R., and William C. Pevec. "Venous thrombosis related to peripherally inserted central catheters." Journal of Vascular and Interventional Radiology 11, no. 7 (2000): 837-840

12. Sajid, Muhammad S., Naeem Ahmed, Mittal Desai, Daryll Baker, and George Hamilton. "Upper limb deep vein thrombosis: a literature review to streamline the protocol for management." Acta haematologica 118, no. 1 (2007): 10-18. Haematol. 2007;118:10-8.

13. Prandoni, Paolo, Enrico Bernardi, Antonio Marchiori, Anthonie WA Lensing, Martin H. Prins, Sabina Villalta, Paola Bagatella et al. "The long term clinical course of acute deep vein thrombosis of the arm: prospective cohort study." Bmj 329, no. 7464 (2004): 484-485.

14. Baarslag, Henk J., Maria MW Koopman, Barbara A. Hutten, May W. Linthorst Homan, Harry R. Büller, Jim A. Reekers, and Edwin JR van Beek. "Long-term follow-up of patients with suspected deep vein thrombosis of the upper extremity: survival, risk factors and post-thrombotic syndrome." European Journal of Internal Medicine 15, no. 8 (2004): 503-507.

15. Becker, Daniel M., John T. Philbrick, and Frederic B. Walker. "Axillary and subclavian venous thrombosis: prognosis and treatment." Archives of internal medicine 151, no. 10 (1991): 1934-1943.

16. Hingorani, Anil, Enrico Ascher, Natalia Markevich, William Yorkovich, Richard Schutzer, Manikyam Mutyala, Suresh Nahata, and Theresa Jacob. "Risk factors for mortality in patients with upper extremity and internal jugular deep venous thrombosis." Journal of vascular surgery 41 , no. 3 (2005): 476-478.

17. Di Nisio, M., G. L. Van Sluis, P. M. M. Bossuyt, H. R. Büller, E. Porreca, and A. W. S. Rutjes. "Accuracy of diagnostic tests for clinically suspected upper extremity deep vein thrombosis: a systematic review." Journal of Thrombosis and Haemostasis 8, no. 4 (2010): 684-692.

18. Stanley, J. M., R. S. McGrath, M. B. Freeman, and S. L. Stevens. "Predictability of symptoms of upper extremity deep venous thrombosis in patients with central venous catheters with color duplex imaging." JOURNAL OF VASCULAR TECHNOLOGY 18 (1994): 71-71.

19. Constans, Joel, Louis-Rachid Salmi, Marie-Antoinette Sevestre-Pietri, Sophie Perusat, Monika Nguon, Maryse Degeilh, Jose Labarere et al. "A clinical prediction score for upper extremity deep venous thrombosis." Thrombosis and haemostasis 99, no. 01 (2008): 202-207.

20. Merminod, Thierry, Sylvie Pellicciotta, and Henri Bounameaux. "Limited usefulness of D-dimer in suspected deep vein thrombosis of the upper extremities." Blood coagulation \& fibrinolysis 17, no. 3 (2006): 225-226.

21. Sullivan, Eugene D., David J. Peter, and John J. Cranley. "Real-time B-mode venous ultrasound." Journal of Vascular Surgery 1, no. 3 (1984): 465-471.
22. Patel, Maneesh C., Laurence H. Berman, Hilary A. Moss, and Simon J. McPherson "Subclavian and internal jugular veins at Doppler US: abnormal cardiac pulsatility and respiratory phasicity as a predictor of complete central occlusion." Radiology 211 , no. 2 (1999): 579-583.

23. Sottiurai, Vikrom S., Kristin Towner, Anne E. McDonnell, and Christopher K. Zarins. "Diagnosis of upper extremity deep venous thrombosis using noninvasive technique." Surgery 91 , no. 5 (1982): 582-585.

24. Haire, William D., Thomas G. Lynch, Gunnar B. Lund, Robert P. Lieberman, and James A. Edney. "Limitations of magnetic resonance imaging and ultrasound-directed (duplex) scanning in the diagnosis of subclavian vein thrombosis." Journal of vascular surgery 13, no. 3 (1991): 391-397.

25. Knudson, Gregory J., Debra A. Wiedmeyer, Scott J. Erickson, W. D. Foley, T. L. Lawson, M. W. Mewissen, and E. O. Lipchik. "Color Doppler sonographic imaging in the assessment of upper-extremity deep venous thrombosis." AJR. American journal of roentgenology 154, no. 2 (1990): 399-403.

26. Baxter, G. M., W. Kincaid, R. F. Jeffrey, G. M. Millar, C. Porteous, and P. Morley "Comparison of colour Doppler ultrasound with venography in the diagnosis of axillary and subclavian vein thrombosis." The British journal of radiology 64, no. 765 (1991): 777-781.

27. Baarslag, Henk-Jan, Edwin JR van Beek, Maria MW Koopman, and Jim A. Reekers. "Prospective study of color duplex ultrasonography compared with contrast venography in patients suspected of having deep venous thrombosis of the upper extremities." Annals of internal medicine 136, no. 12 (2002): 865-872.

28. 28.Baarslag, H. J., E. J. R. Van Beek, and J. A. Reekers. "Magnetic resonance venography in consecutive patients with suspected deep vein thrombosis of the upper extremity: initial experience." Acta Radiologica 45, no. 1 (2004): 38-43.

29. Mukherjee, Dipankar, Charles A. Andersen, Anthony S. Sado, and Michael C. Bertoglio. "Use of light reflection rheography for diagnosis of axillary or subclavian venous thrombosis." The American journal of surgery 161, no. 6 (1991): 651-656.

30. Patwardhan, N. A., F. A. Anderson Jr, B. S. Cutler, and H. B. Wheeler. "Noninvasive detection of axillary and subclavian venous thrombosis by impedance plethysmography." The Journal of cardiovascular surgery 24, no. 3 (1983): 250 .

31. Rodger, Marc A., Susan R. Kahn, Philip S. Wells, David A. Anderson, Isabelle Chagnon, Grégoire Le Gal, Susan Solymoss et al. "Identifying unprovoked thromboembolism patients at low risk for recurrence who can discontinue anticoagulant therapy." Cmaj 179 , no. 5 (2008): 417-426.

32. Eichinger, Sabine, Georg Heinze, Lisanne M. Jandeck, and Paul A. Kyrle. "Clinical perspective." Circulation 121, no. 14 (2010): 1630-1636.

33. Plate, G., E. Einarsson, P. Ohlin, R. Jensen, P. Qvarfordt, and B. Eklöf. "Thrombectomy with temporary arteriovenous fistula: the treatment of choice in acute iliofemoral venous thrombosis." Journal of Vascular Surgery 1, no. 6 (1984): 867-876.

34. Plate, G., E. Einarsson, P. Ohlin, R. Jensen, P. Qvarfordt, and B. Eklöf. "Thrombectomy with temporary arteriovenous fistula: the treatment of choice in acute iliofemoral venous thrombosis." Journal of Vascular Surgery 1, no. 6 (1984): 867-876.

35. Levine, Mark, Michael Gent, Jack Hirsh, Jacques Leclerc, David Anderson, Jeffrey Weitz, Jeffrey Ginsberg et al. "A comparison of low-molecular-weight heparin administered primarily at home with unfractionated heparin administered in the hospital for proximal deep-vein thrombosis." New England Journal of Medicine 334 no. 11 (1996): 677-681.

36. Columbus Investigators. "Low-molecular-weight heparin in the treatment of patients with venous thromboembolism." New England Journal of Medicine 337, no. 10 (1997): 657-662.

37. Kovacs, Michael J., Marc Rodger, David R. Anderson, Beverly Morrow, Gertrude Kells, Judy Kovacs, Eleanor Boyle, and Philip S. Wells. "Comparison of 10-mg and 5 -mg warfarin initiation nomograms together with low-molecular-weight heparin for outpatient treatment of acute venous thromboembolism: a randomized, double-blind, controlled trial." Annals of Internal Medicine 138, no. 9 (2003): 714-719.

38. Harrison, Linda, Marilyn Johnston, M. Patricia Massicotte, Mark Crowther, Karen Moffat, and Jack Hirsh. "Comparison of 5-mg and 10-mg loading doses in initiation of warfarin therapy." Annals of internal medicine 126, no. 2 (1997): 133-136.

39. Martinelli, Ida, Tullia Battaglioli, Paolo Bucciarelli, Serena Maria Passamonti, and Pier Mannuccio Mannucci. "Risk factors and recurrence rate of primary deep vein thrombosis of the upper extremities." Circulation 110, no. 5 (2004): 566-570.

40. Savage, K. J., P. S. Wells, V. Schulz, D. Goudie, B. Morrow, M. Cruickshank, and M. J. Kovacs. "Outpatient use of low molecular weight heparin (Dalteparin) for the treatmen of deep vein thrombosis of the upper extremity." Thrombosis and haemostasis 82 , no. 09 (1999): 1008-1010. 
41. Douketis, James D., Mark A. Crowther, Gary A. Foster, and Jeffrey S. Ginsberg. "Does the location of thrombosis determine the risk of disease recurrence in patients with proximal deep vein thrombosis?." The American journal of medicine 110, no. 7 (2001): 515-519.

42. Brown, Wayne D., and Samuel Z. Goldhaber. "How to select patients with deep vein thrombosis for tPA therapy." Chest 95, no. 5 (1989): 276S-278S.

43. Blinc, A., S. D. Kennedy, R. G. Bryant, V. J. Marder, and C. W. Francis. "Flow through clots determines the rate and pattern of fibrinolysis." Thrombosis and haemostasis 71 , no. 2 (1994): 230 .

44. Blinc, Aleš, and Charles W. Francis. "Transport processes in fibrinolysis and fibrinolytic therapy." Thrombosis and haemostasis 75, no. 04 (1996): 481-491.

45. Meyerovitz, M. F., J. F. Polak, and S. Z. Goldhaber. "Short-term response to thrombolytic therapy in deep venous thrombosis: predictive value of venographic appearance." Radiology 184, no. 2 (1992): 345-348.

46. OTT, PETER, EBBE ELDRUP, PETER OXHOLM, AAGE VESTERGÅRD, and JENS BJERRE KNUDSEN. "Streptokinase therapy in the routine management of deep venous thrombosis in the lower extremities: a retrospective study of phlebographic results and therapeutic complications." Acta Medica Scandinavica 219, no. 3 (1986) 295-300.

47. Gent, Michael, and Robin S. Roberts. "A meta-analysis of the studies of dihydroergotamine plus heparin in the prophylaxis of deep vein thrombosis." Chest 89 , no. 5 (1986): 396S-400S

48. Bounameaux, Henri, J. D. Banga, E. Bluhmki, S. Coccheri, J. N. Fiessinger, W. Haarmann, D. Lockner et al. "Double-blind, randomized comparison of systemic continuous infusion of 0.25 versus $0.50 \mathrm{mg} / \mathrm{kg} / 24 \mathrm{~h}$ of alteplase over 3 to 7 days for treatment of deep venous thrombosis in heparinized patients: results of the European Thrombolysis with rt-PA in Venous Thrombosis (ETTT) trial." Thrombosis and haemostasis 68, no. 03 (1992): 306-309.

49. Francis, Charles W., and Saara Totterman. "Magnetic resonance imaging of deep vein thrombi correlates with response to thrombolytic therapy." Thrombosis and haemostasis 73, no. 03 (1995): 386-391.

50. Protack, Clinton D., Andrew M. Bakken, Nikhil Patel, Wael E. Saad, David L. Waldman, and Mark G. Davies. "Long-term outcomes of catheter directed thrombolysis for lower extremity deep venous thrombosis without prophylactic inferior vena cava filter placement." Journal of vascular surgery 45, no. 5 (2007): 992-997.

51. Enden, T., N-E. KLØW, L. Sandvik, C-E. Slagsvold, W. Ghanima, G. Hafsahl, P. A Holme et al. "Catheter-directed thrombolysis vs. anticoagulant therapy alone in deep vein thrombosis: results of an open randomized, controlled trial reporting on short-term patency." Journal of thrombosis and haemostasis 7, no. 8 (2009): 1268-1275.

52. VVik, Anders, Pål Andre Holme, Kulbir Singh, Eric Dorenberg, Kåre Christian Nordhus, Satish Kumar, and John-Bjarne Hansen. "Catheter-directed thrombolysis for treatment of deep venous thrombosis in the upper extremities." Cardiovascular and interventional radiology 32 , no. 5 (2009): 980-987.
53. 53.Segal, Jodi B., Michael B. Streiff, Lawrence V. Hofmann, Katherine Thornton, and Eric B. Bass. "Management of venous thromboembolism: a systematic review for practice guideline." Annals of internal medicine 146, no. 3 (2006): 211-222.

54. Meissner, Mark H. "Thrombolytic therapy for acute deep vein thrombosis and the venous registry." Reviews in cardiovascular medicine 3, no. S2 (2019): 53-60.

55. Mewissen, Mark W. "Catheter-directed thrombolysis for lower extremity deep vein thrombosis." Techniques in vascular and interventional radiology 4, no. 2 (2001): 111-114.

56. Grewal, Nina K., Jorge Trabal Martinez, Linda Andrews, and Anthony J. Comerota "Quantity of clot lysed after catheter-directed thrombolysis for iliofemoral deep venous thrombosis correlates with postthrombotic morbidity." Journal of vascular surgery 51 , no. 5 (2010): 1209-1214.

57. Kim, Hyun S., Stephen R. Preece, James H. Black, Luu D. Pham, and Michael B. Streiff. "Safety of catheter-directed thrombolysis for deep venous thrombosis in cancer patients." Journal of vascular surgery 47, no. 2 (2008): 388-394.

58. Delomez, Maxence, Jean-Paul Beregi, Serge Willoteaux, Jean-Jacques Bauchart, Bertrand Janne d'Othée, Philippe Asseman, Nessim Perez, and Claude Thery. "Mechanical thrombectomy in patients with deep venous thrombosis." Cardiovascular and interventional radiology 24, no. 1 (2001): 42-48.

59. Molan, Gj Smith, Mp, G. Fitt, and D. M. Brooks. "Mechanical thrombectomy in acute venous thrombosis using an Amplatz thrombectomy device." Australasian radiology 43, no. 4 (1999): 456-460.

60. TTadavarthy, S. Murthy, Philip D. Murray, S. Inampudi, Gwen K. Nazarian, and Kurt Amplatz. "Mechanical thrombectomy with the Amplatz device: human experience." Journal of Vascular and Interventional Radiology 5, no. 5 (1994): 715-724.

61. Kasirajan, Karthikeshwar, Bruce Gray, and Kenneth Ouriel. "Percutaneous AngioJe thrombectomy in the management of extensive deep venous thrombosis." Journal of Vascular and Interventional Radiology 12, no. 2 (2001): 179-185.

62. LLin, Peter H., Wei Zhou, Alan Dardik, Firas Mussa, Panos Kougias, Nasim Hedayati, Joseph J. Naoum, Hosam El Sayed, Eric K. Peden, and Tam T. Huynh. "Catheter-direct thrombolysis versus pharmacomechanical thrombectomy for treatment of symptomatic lower extremity deep venous thrombosis." The American journal of surgery 192, no. 6 (2006): 782-788.

63. Ferris, E. J., W. N. Lim, P. L. Smith, and R. Casali. "May-Thurner syndrome." Radiology 147, no. 1 (1983): 29-31.

64. Patel, Nilesh H., Kenneth R. Stookey, Douglas B. Ketcham, and Andrew H. Cragg. "Endovascular management of acute extensive iliofemoral deep venous thrombosis caused by May-Thurner syndrome." Journal of Vascular and Interventional Radiology 11, no. 10 (2000): 1297-1302.

65. Lamont, Jeffrey P., Gregory J. Pearl, Peter Patetsios, Marc T. Warner, Dennis R. Gable, Wilson Garrett, Bradley Grimsley, Bertram L. Smith, and William P. Shutze. "Prospective evaluation of endoluminal venous stents in the treatment of the MayThurner syndrome." Annals of vascular surgery 16, no. 1 (2002): 61-64.

Copyright: (C2020 Qureshi MN. This is an open-access article distributed under the terms of the Creative Commons Attribution License, which permits unrestricted use, distribution, and reproduction in any medium, provided the original author and source are credited. 